\title{
Luther- und Melanchthon-Autographe in der Württembergischen Landesbibliothek Stuttgart*
}

\author{
Von CARsten KotTManN
}

Im Anschluss an das Jubiläumsjahr der Reformation mag ein Blick darauf gewährt werden, welche eigenhändigen Zeugnisse der Reformatoren in der Württembergischen Landesbibliothek Stuttgart (künftig: WLB) ${ }^{1}$ gelandet sind bzw. sich dort erhalten haben. Dafür sollen die beiden Hauptprotagonisten der von Wittenberg ausgehenden Reformation ab 1517, Martin Luther (1483-1546) und Philipp Melanchthon (1497-1560), und deren Autographe unter den Beständen der WLB zusammengetragen und in den Blick genommen werden. Zu fragen ist, worum es sich bei diesen Stücken handelt und - soweit rekonstruierbar - wie diese eigenhändige Originale der Reformatoren ihren Weg in die WLB gefunden haben. Dieser Beitrag stellt dabei weniger die überlieferten Texte in den Mittelpunkt, da diese

* Folgende Abkürzungen seien eingeführt: CR = Corpus reformatorum, hg. von Karl Gottlieb Bretschneider u.a., 101 Bde., Halle a.d. S.1834-1959, ND New York 1963/64; MBW $=$ Melanchthons Briefwechsel. Kritische und kommentierte Gesamtausgabe, im Auftrag der Heidelberger Akademie der Wissenschaften hg. von Heinz Scheible/Christine Mundhenk, Stuttgart-Bad Cannstatt $1977 \mathrm{ff}$. [die Regesten auch online unter: http://www. haw.uni-heidelberg.de/forschung/forschungsstellen/melanchthon/mbw-online.de.html (18.09. 2016)]; VD16 = Verzeichnis der im deutschen Sprachraum erschienenen Drucke des XVI. Jahrhunderts, hg. von der Bayerischen Staatsbibliothek in München, 26 Bde., Stuttgart 1971-2000 [online unter: http://www.gateway-bayern.de/index_vd16.html (18.09.2016)]; WA $=$ D. Martin Luthers Werke. Kritische Gesamtausgabe (Weimarer Ausgabe). Abteilung 1: Schriften, 73 Bde., Weimar 1883-2009; WA Br = D. Martin Luthers Werke. Kritische Gesamtausgabe (Weimarer Ausgabe). Abteilung 4: Briefwechsel, 18 Bde., Weimar 1930 - 1985. Etliche Digitalisate der WA-Bände sind unter https://de.wikisource.org/wiki/ Martin_Luther (18.09.2016) gelistet. - Für zahlreiche Hinweise habe ich Frau Dr. Christine Mundhenk (Melanchthon-Forschungsstelle der Heidelberger Akademie der Wissenschaften) sehr herzlich zu danken.

${ }^{1} 1765$ gegründet als Herzogliche Öffentliche Bibliothek; ab 1803: Kurfürstliche Öffentliche Bibliothek; ab 1806: Königliche Öffentliche Bibliothek; ab 1901: Königliche Landesbibliothek; ab 1921: Württembergische Landesbibliothek. - Zur Geschichte vgl. grundsätzlich Karl LöFfLER, Geschichte der Württembergischen Landesbibliothek (Beiheft zum Zentralblatt für Bibliothekswesen, Bd.50), Leipzig 1923, ND Nendeln (Liechtenstein)/ Wiesbaden 1968. 
ohnehin weitgehend publiziert sind, sondern fragt nach der Überlieferungsgeschichte und den Provenienzzusammenhängen der Autographen.

Die Luther- und Melanchthon-Autographe sind grundsätzlich auf unterschiedlichen Wegen in die WLB gekommen². Eine dezidierte Sammlungstätigkeit für solchartige Autographe hat die Bibliothek selbt nie an den Tag gelegt und es sind keine (Teil-) Nachlässe der Reformatoren in Stuttgart gelandet. Jedoch trat im 18. Jahrhundert der württembergische Konsistorialdirektor Friedrich Wilhelm Frommann (1707-1787) als Sammler hervor, der unter anderem aus den Akten des Konsistoriums und durch seine Kontakte zu herzoglichen Archivaren die große Menge von 109 Bänden an Autographen zusammentrug. Die Herkunft der einzelnen Stücke ist jedoch im Einzelfall meist nicht mehr nachzuvollziehen ${ }^{3}$. „Leitgedanke der Sammlertätigkeit ist hier die Idee der Repräsentation eines verehrten Menschen durch ein eigenhändiges Schriftstück, ein Gedanke, der von jeher treibende Kraft für das Autographensammeln war. "4 Frommann löste die jeweiligen Autographe aus ihrem historischen Kontext, schnitt Unterschriften aus und entnahm Material aus staatlichen Archiven; diese „zur Reliquie erhobenen Einzelautograph[e]“ klebte er, nach Ständen geordnet und alphabetisch sortiert, in Bände mit Blättern im Kanzleiformat ${ }^{5}$. Auch Stücke von Luther und Melanchthon finden sich in der Frommann-Sammlung; diese stellt damit einen zentralen Überlieferungszusammenhang der in der WLB erhaltenen Autographe der beiden Reformatoren dar.

Unter den Manuscripta der Bibliothek Herzog Friedrichs von WürttembergNeuenstadt, die Ulrich Pregizer 1685 katalogisierte, fanden sich auch Lutheri, et aliorum Theologorum Epistolae, sowie Philippi Melanchthonis variae Epistolae ${ }^{6}$. Die Bibliothek wurde Ende des 17. Jahrhunderts an die württembergische Stammlinie verkauft und gelangte weitestgehend in die Bibliothek des württembergischen

${ }^{2}$ Grundsätzlich zu Luther- und Melanchthon-Autographe vgl. Gustav Koffmane, Die Überlieferung der Werke Luthers, in: Die handschriftliche Überlieferung von Werken D. Martin Luthers. Kritische Untersuchungen, hg. von Gustav Koffmane, Bd.1, Liegnitz 1907, S.III-XXIX, hier S.XIVf.; Otto Albrecht, Kritische Bemerkungen zur Überlieferung der stammbuchartigen Buch- und Bibeleinzeichnungen Luthers, in: Archiv für Reformationsgeschichte 14 (1917) S.161-186; Wilhelm KARG, Wittenberger Stammbucheinträge in der Bayerischen Staatsbibliothek München, in: Zentralblatt für Bibliothekswesen 42 (1925) S. 1-8, hier S. 1; Günther Mecklenburg, Vom Autographensammeln. Versuch einer Darstellung seines Wesens und seiner Geschichte im deutschen Sprachgebiet, Marburg 1963; Werner Wilhelm Schnabel, Das Stammbuch. Konstitution und Geschichte einer textsortenbezogenen Sammelform bis ins erste Drittel des 18. Jahrhunderts (Frühe Neuzeit, Bd.78), Tübingen 2003, S. 244-249.

${ }^{3}$ Vgl. Die Autographensammlung des Stuttgarter Konsistorialdirektors Friedrich Wilhelm Frommann (1707-1787), beschrieben von Ingeborg KreKLER (Die Handschriften der Württembergischen Landesbibliothek, Sonderreihe, Bd. 2), Wiesbaden 1992, S. XXIII.

${ }^{4}$ Krekler, Die Autographensammlung (wie Anm.3) S.XXV.

${ }^{5}$ Ebd., S.XXV und XXVIII.

${ }^{6}$ HStAS J 7 Bü 21. 
Oberrats, aber in einzelnen Fällen auch in die Bibliothek des württembergischen Konsistoriums ${ }^{7}$. Es ist denkbar, dass Frommann auf diesem Weg an einzelne der Luther- und Melanchthon-Autographe gelangt ist.

Über den Umweg Frommann sind einzelne Stücke aus der direkten Kommunikation zwischen Luther bzw. Melanchthon und einer der für die WLB bestandsprägenden Institionen, wie z.B. dem württembergischen Herzogshaus oder Einrichtungen der württembergischen Verwaltung, erhalten geblieben. Eine direkte Kommunikation zwischen Luther bzw. Melanchthon und den württembergischen Herzögen Ulrich (reg. 1498-1519 und 1534-1550) und Christoph (reg. 1550-1568) hat ohnehin nur wenig stattgefunden ${ }^{8}$. Dass sich dennoch eine nicht geringe Zahl an Autographe erhalten hat, ist zu einem Gutteil auch einem gewissen Überlieferungsglück zu verdanken.

Im Folgenden werden die Luther- und Melanchthon-Autographe aufgeführt, die sich in der WLB grundsätzlich auf zwei Arten erhalten haben: Zum einen als Eintragungen in Drucken, vor allem auf Titel- bzw. Vorsatzblättern, und zum anderen als eigenständige Handschriften (hier vor allem als Briefe, aber auch als Entwürfe verschiedener Art) bzw. als Teil einer Handschriften- bzw. Autographensammlung. Die Übersicht, deren einzelne Nummern jeweils mit der heute gütligen Signatur der WLB Stuttgart beginnt, ist durchgezählt und weitgehend chronologisch geordnet. In einem Fall wird ein zweifelhaftes Autograph aufgeführt, da seine Authentizität weder eindeutig verifiziert bzw. falsifiziert ist (Nr. 26), und in einem anderen Fall wird ein Autograph aufgeführt, das nachweislich in der Herzoglichen Öffentlichen Bibliothek vorhanden war, heute aber verloren ist (Nr. 25).

\section{Luther-Autographe}

1. Cod. hist. $2^{\circ} 889-24$, fol. $72^{\text {r }}$ : Überliefert ist der Schluss eines Entwurfs eines Briefes Luthers an Papst Leo X.; dieses Fragment stellt wohl einen frühen Entwurf zu Luthers ersten, im Mai 1518 an den Papst abgesandten Brief dar ${ }^{9}$. Der Entwurf unterscheidet sich erheblich von der endgültigen Abfassung, der letztlich abge-

7 Vgl. Roland Rappmann, Die Bibliothek Herzog Friedrichs von Württemberg-Neuenstadt (1615-1682). Darstellung ihrer Geschichte, masch., Köln 1985, S. 111.

8 Von Luther sind keine Briefe an bzw. von Herzog Ulrich erhalten (vgl. das Korrespondentenverzeichnis in WA Br 16). - Erhalten ist ein Brief Melanchthons an Herzog Ulrich (MBW T16, S. 357 [Nr. 4759]; Regest: MBW 5, S. 123 [Nr. 4769]), und zwischen Melanchthon und Herzog Christoph ist ab 1553 ein kleiner Briefwechsel überliefert (Regesten: MBW 7, S. 45 [Nr. 6765]; MBW 8, S.139f. [Nr. 8388], S. 157 [Nr. 8435], S. 160 [Nr. 8444], S. 165 [Nr. 8457], S. 409 [Nr. 9121] und S. 417 f. [Nr. 9147]). Von Melanchthon verfasst wurde zudem der Brief MBW 8, S. 160 (Nr. 8443).

9 Krekler, Die Autographensammlung (wie Anm.3) S.308. Vgl. WA 9, S.171-173; Abdruck des Fragments ebd., S.173-175; Abb. ebd., Taf.V. - Der Brief vom Mai 1518 in: WA 1, S.527-530; Regest in: WA Br 1, S. 179; deutsche Übersetzung in: Luther deutsch. 
schickte Brief ist in den Formulierungen deutlicher und entschiedener. Das Autograph stammt aus der Sammlung des Konsistorialdirektors Friedrich Wilhelm Frommann (s.o.); das Quartblatt von 20,6 × 14,9 cm Größe wurde auf ein gefenstertes Blatt im Kanzleiformat geklebt, um das auf der Rückseite befindliche weitere Autograph (s. Nr.2) ebenfalls zu erhalten. Es steht zu vermuten, dass Frommann das zweifache Autograph selbst schon in dieser Form erhalten hat. Zur Herkunft dieses Brieffragments ist nichts bekannt.

2. Cod. hist. $2^{\circ}$ 889-24, fol.72v: Dieses Fragment, das das obere Drittel der Rückseite des vorherigen Fragments einnimmt (s. Nr.1), enthält den Entwurf für eine Predigt zum 16. Sonntag nach Trinitatis über Lk 7,11-17 und das Thema der Gerechtigkeit $^{10}$. Angesichts des Überlieferungszusammenhangs mit dem Briefentwurf des Jahres 1518 wird dieser Predigtentwurf in dasselbe Jahr, das heißt auf den 19. September 1518 (=19. Sonntag nach Trinitatis 1518) zu datieren sein. In den Sermones de triplici iustitia von 1518 erwähnt Luther einen früheren Beitrag zum Thema der Gerechtigkeit (Et de ista nunc latius dicendum, ubi dimissum fuit nuper a me), womit vielleicht der hier angezeigte Predigtentwurf gemeint ist ${ }^{11}$.

3. Cod. hist. $2^{\circ} 889-24$, fol. $74^{\mathrm{r}}-\mathbf{7 5}^{\mathrm{v}}$ : Dieser Brief Luthers, ebenfalls aus der Autographensammlung Frommann ${ }^{12}$ und geschrieben am 4. Januar 1526 in Wittenberg, nimmt ausführlich zur Abendmahlsfrage Stellung ${ }^{13}$. Er ist adressiert: $A l$ len lieben Christen Zu Reudlingen meynen lieben Herrn freundenn, brudern ynn Christo. Zwingli hatte dem Reutlinger Reformator Matthäus Alber (1495-1570) kurz zuvor seine Vorstellung des Abendmahls nahegelegt. Da die Reutlinger nun auch Luthers Meinung hören wollten, schickten sie Ende 1525 eine Gesandtschaft nach Wittenberg, auf das der doctor wider Zwingeln schreiben wolle, wie der Wittenberger Theologe Justus Jonas sich ausdrückte ${ }^{14}$ - der hier vorliegende Brief Luthers enthält eine Antwort an die Reutlinger und ist eine Auseinandersetzung mit den Positionen Zwinglis ${ }^{15}$.

Die Werke Martin Luthers in neuer Auswahl für die Gegenwart, Bd.2: Der Reformator, hg. von Kurt Aland, Göttingen ${ }^{2} 1981$, S. 88-91.

10 Krekler, Die Autographensammlung (wie Anm.3) S.308. Vgl. WA 1, S. 203 f.

11 Vgl. WA 1, S. 41-47, hier S. 46.

12 Krekler, Die Autographensammlung (wie Anm. 3) S. 308.

13 WA 19, S.114-125; zur Handschrift ebd., S.117; Edition des Briefs nach der Handschrift ebd., S.118, 120, 122, 124; Matthäus Alber, Reformator von Reutlingen. Berichte und Dokumente, hg. von Christoph Duncker, Weinsberg 1971, S. 10-13, Abb. S. 18 -21 (fälschlich mit Angabe: „Original beim Hauptstaatsarchiv Stuttgart“); Nachdruck: Martin Luther, Brief an die Reutlinger vom 4. Januar 1526, Nürnberg 1980.

${ }^{14}$ Zitiert nach WA 19, S.115 f.

15 Vgl. WA 19, S. 114-116; Martin BRECHT, Reutlingen und die Reformation in Deutschland, in: BWKG 80/81 (1980/81) S. 5-23, hier S. 15 f. 
Nachträglich wurden in Luthers Autograph nahezu alle diakritischen Zeichen von anderer Hand hinzugefügt ${ }^{16}$. Die sonst eigenhändige Abfassung wurde bereits wohl im 17. Jahrhundert durch den Nachtrag am Ende des Briefs mit den Worten Original-Schreiben $H$ [errn] $D$ [omi]ni $D$ [octo]ris Martini Lutheri vermerkt. Am unteren Ende der letzten Seite findet sich noch eine Wiederholung der Datumsangabe: [D]onnerstags nach dem Neuen Jabrs tag Anno 1526. Darunter: Num[ero] 18., was sich wohl auf eine archivalische oder bibliothekarische Signatur bezieht. Auf welchen Wegen der Brief in die Sammlung Frommann gelangte, ist nicht bekannt.

4. Cod. hist. $4^{\circ}$ 95: Dieses Konvolut überlieferte ursprünglich mehrere Originalbriefe Luthers sowie jeweils einen autographen Entwurf von Melanchthon (s. Nr.33) und einen autographen Brief des Tübinger Gräzisten Martin Crusius $(1526-1607)^{17}$. Von den im Katalog der Stuttgarter historischen Handschriften von Wilhelm von Heyd genannten Lutherbriefen handelt es sich bei Nr.1.b („an einen Unbekannten ohne Anfang und Ende (deutsch)“) wohl um ein Blatt der heutigen Signatur Cod. theol. et philos. $2^{\circ} 300$ (s. Nr. 8). Zudem nennt von Heyd eine „Epistola Lutheri ad Matthaeum Alberum, evangelistam Reutlingensem (Orig.), welche früher in diesem Faszikel lag“, und die „seit 1866 vermisst“ wird - es scheint unwahrscheinlich, dass es sich dabei um den Brief Luthers an Matthäus Alber vom 4. Januar 1526 drehen könnte, da dieser Teil der Frommann'schen Sammlung ist und dort nicht erst nachträglich hinzugefügt wurde. Somit ist der einzige autographe Lutherbrief, den Cod. hist. $4^{\circ} 95$ bis heute enthält, ein Schreiben an den Coburger Pfarrer Johann Fesel (Feselius, $†$ 1557) vom 5. Oktober 1530, geschrieben in Neustadt bei Coburg ${ }^{18}$. Fesel, der wohl in Wittenberg studiert und dort die engere Bekanntschaft zu Melanchthon gemacht hatte, war vermutlich bereits 1525 Pfarrer der Heilig-Kreuz-Kirche in Coburg ${ }^{19}$; mit Luther stand er nachweislich zwischen Oktober 1530 und April 1531 im Briefkontakt ${ }^{20}$. Das hier vorliegende Autograph befand sich 1753/1754 in der württembergisch herzoglichen Kunst-

16 Vgl. WA 19, S. 118.

17 Die Historischen Handschriften der Königlichen öffentlichen Bibliothek, beschrieben von Wilhelm von HeYd, Bd.2 (Die Handschriften der Königlichen öffentlichen Bibliothek zu Stuttgart, Bd.1,2), Stuttgart 1891, S.38f.

18 WA Br 5, S.649f. (Nr. 1734). Wilhelm von Heyd (Die Historischen Handschriften der Königlichen öffentlichen Bibliothek, Bd.2 [wie Anm.17] S.38) nannte irrtümlich das Abfassungsjahr 1537.

${ }_{19}$ Vgl. Georg Paul Hönn, Sachsen-Coburgische Chronik. In welcher eine genaue Beschreibung aller in den Fürstenthümern S. Coburg und S. Hildburghausen gelegenen Ortschaften und Klöstern, nebst alles dessen, was sich seit dem Jahre 741 bis 1792 in Friedensund Kriegszeiten, in geist- und weltlichen Dingen etc. in diesen Fürstenthümern ereignet, enthalten ist ..., Bd.2, Coburg 1806, S. 354 f.; Pfarrerbuch der Pflege Coburg, bearb. von Albert GreInER, masch., [Tübingen] 1944, S. 150.

20 Weitere Briefe in: WA Br 5, S.694; WA Br 6, S. $84 \mathrm{f}$. 
kammer: Schreiben $D$ [octor] Luthers de $A[\mathrm{nn}]$ o 1531 [!] 5.t[en] Oct[obris] an einen Coburgischen Pfarrer M[agister] Joh[annes] Fuselius [! $]^{21}$. Danach kam es in das württembergische Naturalienkabinett, dessen handschriftlicher Bestand 1776 nach dem Umzug der Herzoglichen Öffentlichen Bibliothek von Ludwigsburg nach Stuttgart in deren Sammlung integriert wurde ${ }^{22}$.

5. R 16 Lut 22: Das Stuttgarter Exemplar des um 1530 im Druck erschienenen Briefs Martin Luthers an den Erzbischof von Mainz, Albrecht Kardinal von Brandenburg (1490-1545) ${ }^{23}$ trägt auf dem Titelblatt Luthers Widmung Magistro Johanni Weybringer parocho Heylburghausen \| M[artin] Luther.

1529 erhielt Johann Weybringer auf Empfehlung von Luther die Pfarrstelle in Hildburghausen (heute zu Thüringen) ${ }^{24}$. Weybringer wurde am 4. März 1528 als Joannes Weybringer [...] de landshut unter den pauperes gratis recepti an der Universität Wittenberg immatrikuliert ${ }^{25}$ und stand dort wohl in einem engeren Verhältnis zu Luther, den er seinen Praeceptor und Patron nannte ${ }^{26}$. Bereits 1530 wandte sich Weybringer erneut an Luther „infolge der wenig erfreulichen Amtserfahrungen, die er mit der Bevölkerung der Stadt Hildburghausen [...] gemacht

${ }^{21}$ HStAS A 20 a Bü 37, $7^{\mathrm{r}}\left[4^{\mathrm{r}}\right]$.

22 WLB Stuttgart, Bibliotheksakten, Nr.14: Ein eigenbändiges Lateinisches Schreiben von D[octor] Martin Luther, an M[agistrum] Johann Fuselium [!], Pfarrer in Coburg, $d[\mathrm{e}]$ $d$ [it] 5. Oct[obris] 1531 [!]. (unter Manuscripta, Nr. 6); Löffler, Geschichte (wie Anm.1) S.15; Carsten KotTmann, Die Bibliothek des herzoglich württembergischen Naturalienkabinetts in der zweiten Hälfte des 18. Jahrhunderts, in: Bibliothek. Forschung für die Praxis. Festschrift für Konrad Umlauf zum 65. Geburtstag, hg. von Petra Hauke/Vivien Petras/Andrea Kaufmann, Berlin 2017, S. 589-603, hier S. 595 u. 600.

${ }^{23}$ Martin Luther, Ein Brieff an den Cardinal Ertzbischoff zu Mentz, [Nürnberg]: [Johann Petreius für Georg Rottmaier], [1530] (VD16 L 4130). - Digitalisat unter http://digital. wlb-stuttgart.de/purl/bsz351431594 (18.09.2016).

${ }^{24}$ Johann Werner Krauss, Beyträge zur Erläuterung der Hochfürstl. Sachsen-Hildburghäusischen Kirchen- Schul- und Landes-Historie. Aus bewährten Urkunden gesammlet und nebst einer Vorrede und der Einrichtung des gantzen Wercks, wie auch am Ende mit beygefügten dreyfachen Register über die in allen Theilen fürkommende Personen, Oerter und Sachen, Bd. 2: Von der Stadt und Dioeces Hildburghausen, Greiz im Vogtland 1752, S. 199-203; DERs., Antiquitates Et Memorabilia Historiae Franconicae, Darinnen insonderheit der Ursprung, Einrichtung und Merckwürdigkeiten der Fürstlichen Residenz-Stadt Hildburghausen von denen ältesten biß auf die jetzige Zeiten aus bewährten Uhrkunden abgehandelt werden, Hildburghausen 1753, S.199-203; HönN (wie Anm. 19) S.344; Gottlieb Јасов, Namensverzeichnis der Studierenden des Herzogtums S. Meiningen, welche in der Zeit von 1502-1560 die Universität Wittenberg besuchten, in: Schriften des Vereins für Sachsen-Meiningische Geschichte und Landeskunde 20 (1885) S. 53-70, hier S. 54 Anm. 98. Zum Hintergrund vgl. Georg Berbig, Luther-Urkunden aus Coburg und Gotha, in: Zeitschrift für Kirchengeschichte 21 (1901) S.139-148, hier 144f. Der zugrundeliegende Briefwechsel in WA Br 5, S.647-649 (Nr.1732f.).

${ }_{25}$ Album Academiae Vitebergensis, Bd.1: ab a. Ch. MDII usque ad a. MDCLX, hg. von Karl Eduard Foerstemann, Leipzig 1841, ND Magdeburg 1976, S. 134.

26 Krauss (wie Anm. 24) S. 200. 
hatte“27; er traf ihn am Tag der Abreise Luthers von der Veste Coburg persönlich an, und Luther gab ihm ein weiteres Empfehlungsschreiben mit, das die Situation in Hildburghausen wohl offensichtlich verbesserte ${ }^{28}$.

Luther versorgte Weybringer für die Stelle in Hildburghausen mit einigen seiner Texte, die er jeweils mit einer Widmung versah. Darunter war der Band Der Prophet Daniel Deudsch in der Luthers Übersetzung, gedruckt 1530 bei Hans Lufft in Wittenberg ${ }^{29}$, der sich bis heute in der Bibliothek der Christuskirche in Hildburghausen befindet ${ }^{30}$. Drei weitere Bände stammen aus der Sammlung des Schriftstellers und Bibliothekars in Meiningen Ludwig Bechstein (1801-1860). Zwei dieser Bände (Martin Luther, Ein Widderruff vom Fegefeur, Wittenberg: Georg Rau, 1530 [VD16 L 7544]; Ein sendbrief D. M. Luthers. Vom Dolmetzschen und Fürbit der Heiligenn, [Nürnberg]: Georg Rottmaier, 1530 [VD16 L 5950]), befinden sich heute in Philadelphia (PA) ${ }^{31}$.

Das Stuttgarter Exemplar von Luthers Brief-Druck an den Mainzer Erzbischof Albrecht von Brandenburg stammt aus der Herzoglichen Sachsen-Meiningischen Bibliothek; in ihm findet sich auf dem Vorsatzblatt eine kurze Notiz von der Hand Ludwig Bechsteins über die Hintergründe der Widmung Luthers an Weybringer. In die WLB kam der Band erst nach 1969, wie der Quadratstempel zeigt ${ }^{32}$; im Jahr 1927 befand sich der Band offensichtlich noch in Privatbesitz ${ }^{33}$.

6. Cod. hist. $2^{\circ} 889-24$, fol. $73^{\text {r }}$ : Dieses Autograph ist undatiert und enthält als kurze Notiz eine ironische Kritik Luthers an der päpstlichen Kurie. Dieser Zettel $(7,5 \times 13,5 \mathrm{~cm})$, der in der Frommann'schen Autographensammlung (s. Nr.1) überliefert ist ${ }^{34}$, wurde auf ein Blatt in Kanzleiformat aufgeklebt, auf dem in der Mitte ein Portrait Luthers gemalt ist $(8,5 \times 7 \mathrm{~cm}$; Luther im Talar, vor sich eine aufge-

27 Berbig (wie Anm. 24) S. 144.

${ }^{28} \mathrm{Zu}$ Weybringer vgl. auch WA 48, S. 253 und 679 Anm. 4.

29 VD16 B 3828.

30 Felicitas Marwinski/Karl-Heinz Ross, Art. [Hildburghausen], Bibliothek der Christuskirche, in: Handbuch der historischen Buchbestände in Deutschland, Bd.20, hg. von Friedhilde Krause/Felicitas Marwinski, Hildesheim 1999, S. 33.

31 University of Pennsylvania Philadelphia, Rare Books and Manuscripts Library, 838 L97V2 und 838 L97Wi. Vgl. Rudolf Hirsch, Suo Hospiti Dono Dedit, in: The Library Chronicle of the Friends of the Library of Pennsylvania 33,1 (1957) S.35. Eine zeitgenössische Nachzeichnung einer dieser Widmungen befindet sich heute im Kestner-Museum Hannover, vgl. WA 48 S.253f. Anm. a.

32 Vgl. Bibliotheksstempel. Besitzvermerke von Bibliotheken in der Bundesrepublik Deutschland, hg. von Antonius Jammers (Beiträge aus der Staatsbibliothek zu Berlin, PreuBischer Kulturbesitz, Bd.6), Wiesbaden 1998, Nr.130.

33 Vgl. WA 48, S. 254.

34 Vgl. WA 9, S.171; Otto Albrecht, Zwei versprengte Konzepte Luthers, aus den Originalen zum erstenmal veröffentlicht, in: Theologische Studien und Kritiken 96/97 (1925) S.318-322, hier S.318f. (mit Textabdruck); KreKLer, Die Autographensammlung (wie Anm.3) S.308. 
schlagene Bibel, die linke Hand unter dem Vorderdeckel, die rechte Hand auf der aufgeschlagenen rechten Seite). Zwischen der Notiz und dem Portrait befindet sich, ebenfalls aufgeklebt, ein Papierstreifen wohl des 17. Jahrhunderts: Quae sequuntur scripta sunt manu $D$ [octoris] $M$ [artini] $L$ [utheri]. Der Schrift nach könnte die Notiz in die 1530 er Jahre zu datieren sein. Auf welchen Wegen die Notiz in die Sammlung Frommanns kam, ist unbekannt.

7. Cod. hist. $2^{\circ} \mathbf{8 8 9 - 2 4}$, fol. $\mathbf{7 6}^{\mathrm{rv}}$ : Hier notierte Luther, wohl sehr eilig und ungeordnet, die Merkmale der päpstlichen Kirche und der evangelischen Lehre. Auch dieses Konzept stammt wohl aus den 1530er Jahren ${ }^{35}$. Otto Albrecht vermutete darin eine Vorarbeit zu Luthers „Vermahnung an die Geistlichen, versammelt auf dem Reichstag zu Augsburg“ von $1530^{36}$ bzw. Luthers „Rhapsodia seu Concepta

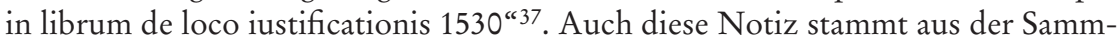
lung Frommann; die Notiz in der Größe von 21,5 x $17 \mathrm{~cm}$ wurde auf ein gefenstertes Blatt im Kanzleiformat geklebt. Weitere Hinweise auf eine frühere Provenienz liegen nicht vor.

8. Cod. theol et philos. $2^{\circ}$ 300: Dieses Konzept Luthers einer Ostermontagspredigt (früher Stuttgart, WLB, Cod. hist. $4^{\circ} 95,1 \mathrm{~b}$ ) ${ }^{38}$ wurde vor 1544 niedergeschrieben und ist lediglich als Fragment auf einem Doppelblatt erhalten. Die Predigten nach dem Kirchenjahr, zu denen diese Ostermontagspredigt gehört, wurden 1544 von Hans Lufft in Wittenberg gedruckt ${ }^{39}$. Zahlreiche Korrekturen und Durchstreichungen heben den Konzeptcharakter des Autographs hervor, das die einzig erhaltene Handschrift zur Druckausgabe durch Hans Lufft von 1544 darstellt. Diese Ausgabe wurde von Caspar Cruciger betreut; das Autograph enthält Nach-

35 Vgl. WA 9, S.171; Albrecht (wie Anm.34) S.319-322 (mit Textabdruck); Krekler, Die Autographensammlung (wie Anm.3) S. 308.

36 WA 30 II, S. 237-356. Vgl. Freiheit - Wahrheit - Evangelium. Reformation in Württemberg. Katalog zur Ausstellung des Landesarchivs Baden-Württemberg, Hauptstaatsarchiv Stuttgart, bearb. von Peter RüCKERT, Ostfildern 2017, S. 74 f.

37 WA 30 II, S. 657-676.

38 Die Historischen Handschriften der Königlichen öffentlichen Bibliothek, Bd. 2 (wie Anm. 17) S. 39. Vgl. zukünftig: Codices theologici et philosophici der Neuzeit, beschrieben von Carsten Kottmann, Wiesbaden 2018 (Die Handschriften der Württembergischen Landesbibliothek Stuttgart 1,7) [im Druck].

${ }^{39}$ Martin Luther, Auslegung der Episteln und Euangelien/von Ostern bis auff das Advent ... Auffs new zugericht, [hg. von Caspar CRUCIger], Wittenberg: Hans Lufft, 1544 (VD16 L 5610). Die Ostermontagspredigt ist dort gedruckt auf fol. XVIIv, Zeile 19 bis fol. XVIII", Zeile 4. Kritische Edition in: WA 21, S. 227, 32-229, 29 (nicht nach dieser Handschrift, sondern nach dem Druck); WA 22, S. 434-437 (nach dieser Handschrift, mit Faksimile). Vgl. auch Ernst Thiele, Die Originalhandschriften Luthers, in: Lutherstudien zur 4. Jahrhundertfeier der Reformation, veröffentlicht von den Mitarbeitern der Weimarer Lutherausgabe, Weimar 1917, S. 259; WA Br 14, S.133f. 
träge von dessen $\mathrm{Hand}^{40}$. Somit befand sich die Handschrift wohl zu Zeiten des Drucks in Crucigers Besitz. Vermutlich kam sie 1776 im Zuge der Inkorporierung zahlreicher kleinerer Stuttgarter Behördenbibliotheken in die Herzogliche Öffentliche Bibliothek ${ }^{41}$ aus der Bibliothek des Herzoglich württembergischen Naturalienkabinetts; in dessen Abgabeverzeichnis ist gelistet: $D$ [octor] Martin Luthers eigene Handschrifft, teutsch, in 2. folio - aber weil sie zerrißen, in 4. Quart Blättlein ${ }^{42}$. Im Jahr 1753/1754 befand sich dieser Autograph in der Handschriftensammlung der württembergischen Kunstkammer: $D$ [octor] Martin Luthers eigene Handschrifft auf 4. quart Blättlin ${ }^{43}$. Die hier genannten quart Blättlin wurden später wieder zusammengeklebt; die Klebesteifen sind heute noch erhalten. Danach wurden die beiden entstandenen folio-Blätter auf zwei unterschiedliche Signaturen aufgeteilt, bis die Handschrift zum einen aus ehemals Stuttgart, WLB, Cod. hist. $4^{\circ}$ $95,1 \mathrm{~b}^{44}$ und zum anderen aus einem Blatt aus ehemals Stuttgart, WLB, Cod. theol. et philos. $4^{\circ} 272$ nach 1891 in der heutigen Signatur Cod. theol. et philos. $2^{\circ} 300$ wieder zusammengefügt wurde.

Dem Fragment beigefügt ist eine Postkarte des evangelischen Theologen Georg Buchwald (1859-1947) ${ }^{45}$ als Mitglied der Kommission zur Herausgabe der Werke Martin Luthers an die WLB, geschrieben am 29. November 1928 in Rochlitz (Sachsen), mit Hinweisen zur Überlieferung und zum Druck des Texts der Handschrift, sowie vier photomechanische Reproduktionen zur Verwendung für die Edition im 22. Band der Weimarer Ausgabe von Luthers Werken.

9. B griech. 154001: In einen Druck des griechischen Neuen Testaments, Basel: Thomas Platter d. Ä./Robert Winter, 1540 (VD16 B 4182) ${ }^{46}$ trug Luther im Jahr 1545 auf den vorderen Spiegel unter der gleichzeitigen Notiz Si quis graecae linguae usum comparare vult ... eines unbekannten Schreibers eine Bemerkung zu Röm 1,17 (Iustus ex fide sua vivet, ursprünglich aus Hab 2,4) an einen unbekannten Empfänger ein ${ }^{47}$. Unter Luthers Inskription findet sich eventuell ebenfalls aus dieser Zeit der auf dem Kopf stehende Eintrag dıєєoos (?), der ein Hinweis auf die Provenienz des Bandes im 16. Jahrhundert sein könnte. Für das 18. Jahrhundert ist

40 WA 22, S. XII, hier Anm. 4.

41 Löfrler, Geschichte (wie Anm.1) S. 15.

42 WLB Stuttgart, Bibliotheksakten, Nr. 14 (unter Manuscripta, Nr.7). Vgl. KotTmann, Die Bibliothek (wie Anm. 22) S. 595 u. 600.

${ }^{43}$ HStAS A 20a Bü 37, $7^{\mathrm{r}}\left[4^{\mathrm{r}}\right]$.

${ }^{44}$ Vgl. Die Historischen Handschriften der Königlichen öffentlichen Bibliothek, Bd.2 (wie Anm.17) S.39

${ }^{45} \mathrm{Vgl} . \mathrm{zu}$ ihm NDB 2 (1955) S. $710 \mathrm{f}$.

46 Digitalisat unter: http://digital.wlb-stuttgart.de/purl/bsz35126888X (18.09.2016).

47 Vgl. WA 48, S. 200 (Nr.268). - Griechische Bibeldrucke, beschrieben von Stefan Strohm unter Mitarbeit von Peter Amelung/Irmgard Schauffler/Eberhard Zwink (Die Bibelsammlung der Württembergischen Landesbibliothek Stuttgart, Bd.1,3), Stuttgart-Bad Cannstatt 1984, S.21 (C 32). 
die Besitzgeschichte deutlich besser belegt: Nach der Verlegermarke des Johann Schabler auf der letzten Seite ${ }^{48}$ befindet sich auf dem hinteren Spiegel das Wappen-Exlibris des Pfarrers, Gießener Theologieprofessors und Superintendenten Johann Gottfried Schupart $(1677-1730)^{49}$, sowie darunter der Eintrag Hunc librum nunc jure possidet Johannes Laur[entius] Frid[ericus] Schupart a[nno] MCCLXI. von dessen Enkel Johann Lorenz Friedrich Schupart (1745-1764), der als Primaner und Contubernalis 18-jährig starb. Wahrscheinlich gelangte der Band über Johann Gottfrieds Sohn, August Gottfried Schupart, in den Besitz des Enkels ${ }^{50}$. Wie der Band schließlich in die Herzogliche bzw. Königliche Öffentliche Bibliothek gelangte, ist unklar; laut Stempel müsste es aber spätestens zwischen ca. 1810 und 1862/1863 geschehen sein.

10. R 16 Lut 1: Dieser Band (ehemals Theol.oct.20097) beinhaltet zwei Werke Martin Luthers (Enchiridion Der kleine Catechismus. Für die gemeine Pfarherr und Prediger, Leipzig: Valentin Bapst d.Ä., 1544 [VD16 L 5060]; Ein einfeltige weise zu beten, fur einen guten freund, Leipzig: Valentin Bapst d. Ä., 1544 [VD16 L 4502]) sowie den Druck des Urbanus Rhegius, „Seelen-Ertzney fur die Gesunden und Krancken in Tods-nöten, Leipzig: Valentin Bapst d. Ä., 1545“ (VD16 R 1913) und den dort begefügten anonym überlieferten Druck „Vom glauben und guten wercken. Etliche sprüche vom glauben aus dem Alten und Newen Testament, Leipzig: Bapst, 1545“ (VD16 V 2363). Auf dem vorderen Spiegel befindet sich ein autographer Eintrag Luthers, eine Übersetzung von Mt 7,7f.: Betet, So werdet yhrs kriegen // Suchet, So werdet ybrs finden // Klopft an, So wird euch auffgethan // Denn wer da betet, der kriegt // Wer da sucht, der findet // Wer anklopfet, dem wirt auffgethan. // Martinus Luther D[octor] // 1545 51 . Dieser Wortlaut der Übersetzung von Mt 7,7f. findet sich in keiner Bibelausgabe Luthers; was

48 Vgl. Josef Benzing, Die deutschen Verleger des 16. und 17. Jahrhunderts. Eine Neubearbeitung, in: Archiv für Geschichte des Buchwesens 18 (1977) Sp.1078-1322, hier Sp. 1291.

49 Vgl. Herzog August Bibliothek Wolfenbüttel, Berlepsch Exlibris Teil I, S. 213, Nr. 248 (http://diglib.hab.de/?grafik=exlib-berlepsch-18-2-00250, 18.09.2016); Friedrich WARNECKE, Die deutschen Bücherzeichen (Ex-Libris) von ihrem Ursprung bis zur Gegenwart, Berlin 1890, S. 189 (Nr. 1978). - Zu Schupart vgl. ADB 33 (1891) S. 65-67; Kraichgau-Odenwald, Teil 2: Die Pfarrer und Lehrer der höheren Schulen von der Refomation bis zum Beginn des 19. Jahrhunderts, bearb. von Max-Adolf Cramer (Baden-Württembergisches Pfarrerbuch, Bd. I,2), Karlsruhe 1988, Nr. 3275.

50 Vgl. Pfarrerbuch Württembergisch Franken, Teil 2: Die Kirchen- und Schuldiener, bearb. von Otto Haug unter Mitarbeit von Max-Adolf Cramer und Marlene Holtzmann (Baden-württembergisches Pfarrerbuch, Bd.II,2), Stuttgart 1981, Nr. 2427.

51 Dieser und die folgenden Einträge sind vollständig gedruckt bei Theodor Friedrich Sснотт, Ein Autographon von Luther und Melanchthon, in: Theologische Studien und Kritiken 69 (1896) S.162-164; Otto Albrecht, Zur Bibliographie und Textkritik des Kleinen Lutherschen Katechismus, in: Archiv für Reformationsgeschichte 1 (1903/04) S. 247-278, hier S.275; WA 48, S. 107 (Nr. 143); vgl. auch WA 30,1, S. 685. 
aber grundsätzlich - da er die Zitate häufig aus dem Gedächtnis anführte - vorkam, nicht nur in solchen Bucheinzeichnungen, sondern auch in seiner schriftstellerischen Tätigkeit ${ }^{52}$. Auf der Vorder- und Rückseite des Vorsatzblattes findet sich auch ein Autograph Philipp Melanchthons (s. Nr.22).

Luther hatte den Band, der noch original eingebunden ist, Eva Rosina Starck, geborene von Stickhöllberg, zugeeignet: Dises buch hadt der Godsellig man, Dochkbtor Marthin Ludter, Meiner Frau Andl [Großmutter] Franen Rossina Ein Gebornne Von Stickhölberg zu gudter gedachtnuß verertt (nach Titelblatt). Den Eintrag schrieb 1619 der Enkel der Eva Rosina, Matthäus Starck zusammen mit seiner Frau Anna Maria Starck, geborene von Birnbaum (Pirnpaumb) eigenhändig unter das Luther-Autograph. Bereits 1572 trug Ferdinand Starck, Röm[isch] khay[serlichen] May[estät] Dienner, seinen Namen eigenhändig unter die Luther-Verse auf dem vorderen Spiegel ein; dabei handelt es sich vielleicht um den Sohn der Eva Rosina Starck. Der Band blieb im Anschluss zwei weitere Generationen in Familienbesitz und kam in den Besitz des Enkels von Matthäus Starck selbst: des aus Niederungarn stammenden Johann Kaspar Kessler, ab 1675 Oberrats-Skribent, -Vizesekretär und Kreiskanzlist sowie ab 1683 Organist der Stuttgarter und Stiftskirche ${ }^{53}$. Kessler war Taufpate des württembergischen Archivars und Hofregistrators Philipp Jakob Zeitter $(1634-1691)^{54}$, dem er den Band weiterschenkte. Dies geht aus dem Besitzeintrag Zeitters hervor: Dieses Ur-AltVäter- und Mütterliche Andencken verehrt seinem hochwerthesten Herrn Freund und lieben Gevattern: Herrn Philipp Jacob Zeittern, Fürstl[ich] Württemb[ergischen] woblverdienten Archivario. Johann Caspar Kesler, als ein UrEnckel und wabrhafftiger gewester Possessor (nach Titelblatt), aus dem Jahr 1686. Aus dem Besitz des Archivars Zeitter kam der Band wohl in eine der Bibliotheken innerhalb der württembergischen Verwaltung und von dieser dann wohl 1776 in die Herzogliche Öffentliche Bibliothek.

11. Cod. hist. $2^{\circ}$ 527: Die Eigenhändigkeit dieses Briefs Luthers an Philipp Melanchthon vom 1. Februar $1546^{55}$, geschrieben wenige Tage vor Luthers Tod während der Verhandlungen mit den Grafen von Mansfeld in Eisleben ${ }^{56}$, wurde

52 Vgl. Hans Volz, Die Bibeleinzeichnungen der Wittenberger Reformatoren. Eine buchgeschichtliche literarhistorische Studie, in: Gutenberg-Jahrbuch 46 (1971) S.122-137, hier S. 123.

53 Walther Pfeilsticker, Neues württembergisches Dienerbuch, Bd.1, Stuttgart 1957, \891, 954, 1237, 1260, 1339, 1386; Robert EITNER, Biographisch-Bibliographisches Quellen-Lexikon der Musiker und Musikgelehrten der christlichen Zeitrechnung bis zur Mitte des neunzehnten Jahrhunderts, Bd. 5, Leipzig 1901, S. 355.

${ }^{54}$ Pfeilsticker (wie Anm. 53) $\$ 1174,1281,1388,2053$.

${ }^{55} \mathrm{Vgl}$. Die Historischen Handschriften der Königlichen öffentlichen Bibliothek, beschrieben von Wilhelm von HEYD, Bd.1 (Die Handschriften der Königlichen öffentlichen Bibliothek zu Stuttgart, Bd.1,1), Stuttgart 1890, S. 224.

${ }^{56}$ Martin Brecht, Martin Luther, Bd. 3: Die Erhaltung der Kirche. 1532-1546, Stuttgart 1987, S. 362-368. 
zwar vom Luther-Herausgeber Otto Clemen im Jahr 1948 in Zweifel gestellt, allerdings nicht an Hand des Schriftbilds (da ihm das Original nicht vorlag), sondern auf Grund unüblicher Abkürzungen ${ }^{57}$. Seit dem 1970 von Hans Volz und Eike Wolgast herausgegebenen „Verzeichnis der Lutherbriefhandschriften“ kann der Brief aber mit Sicherheit als Autograph gelten ${ }^{58}$.

Der Lutherbrief befindet sich in einer Sammelmappe, die zusätzlich noch autographe Briefe Melanchthons (s. Nr. 29), Johannes Brenz' (1499-1570), Pietro Paolo Vergerios (1498-1565), Veit Dietrichs (1506-1549), Johannes Calvins (1509-1564) und Johannes Ecks (1486-1543) enthält. Vermutlich befanden sich die Briefe bereits 1688 in der Kunst- und Rüstkammer des Benediktinerklosters Weingarten, wo Johann Ulrich Pregizer seltene epistolae manuscriptae [...] von Philippo Melanchthone und Petro Paulo Vergerio ausmachte; diese vermutete er aus dem Besitz des Schlosshauptmanns auf Hohentübingen Nikolaus Ochsenbach (1562-1626) ${ }^{59}$, dessen Bibliothek mit dem Nachlass seines Sohnes Johann Friedrich Ochsenbach (1606-1658) nach Weingarten $\mathrm{kam}^{60}$. Von Weingarten gelangten die Briefe nach 1810 im Zuge der Säkularisation in die damalige Königliche Öffentliche Bibliothek $^{61}$.

\section{Melanchthon-Autographe}

12. Cod. hist. $2^{\circ} \mathbf{8 8 9}-\mathbf{2 6}$, fol.31 ${ }^{\mathrm{r}}$ : Auf dieser Seite schrieb Melanchthon ein Schema zum Begriff Homo. Von späterer Hand wurde darunter der autographe Charakter der Notiz vermerkt sowie seine Entstehung in Wittenberg verortet und in das Jahr 1519 datiert. Das Blatt stammt aus der Autographensammlung des Friedrich Wilhelm Frommann.

57 WA Br 11, S. 277-279 (Nr. 4196), dazu WA Br 13, S. 352 f.

58 Vgl. WA Br 14, S. 134 (Nr. 294); so auch MBW T15, S. 66 f. (Nr. 4134); MBW 4, S. 319 (Nr. 4134; Regest).

${ }_{59} \mathrm{Zu}$ ihm vgl. Die Handschriften der ehemaligen Hofbibliothek Stuttgart, Bd. 5: Codices Wirtembergici (HB XV 1-127). Codices militares (HB XVI 1-2), beschrieben von Magda Fischer (Die Handschriften der Württembergischen Landesbibliothek Stuttgart, Bd.2,5), Wiesbaden 1975, S. $92-95$.

60 Joseph A. Giefel, Johann Ulrich Pregitzers Reise nach Oberschwaben im Jahr 1688, in: WVjH 11 (1888) S. 36-49, hier S. 45; Die Handschriften der ehemaligen Hofbibliothek Stuttgart, Bd. 5 (wie Anm. 59) S.100; Georg Wilhelm ZAPF, Reisen in einige Klöster Schwabens, durch den Schwarzwald und in die Schweiz. Im Jahr 1781. Worinn von Bibliotheken, Alterthümern, geschichte und vom Zustand der Litteratur überhaupt Nachricht gegeben wird, Erlangen 1786, S.221-230.

${ }^{61}$ Vgl. Karl Löffler, Die Handschriften des Klosters Weingarten (Zentralblatt für Bibliothekswesen, Beiheft 41), Leipzig 1912, S. 144 (Nr. 36). 
13. Cod. hist. $2^{\circ} 889-26$, fol. $36^{\text {rv }}$ : Am 4./5. Oktober 1529 schrieb Melanchthon vom Marburger Religionsgespräch einen Brief an den Vikar Johannes Butzbach, den Reformator Matthäus Alber und den Pfarrer Martin Reiser, alle in Reutlingen, der in der Frommann'schen Sammlung autograph erhalten ist ${ }^{62}$. Friedrich Wilhelm Fromman, aus dessen Autographensammlung der Brief stammt, erhielt diesen sowie die beiden weiteren Briefe Melanchthons an Matthäus Alber (s. Nr. 16 und 17) wohl aus einer gemeinsamen Provenienz. Alle drei Stücke tragen den Eintrag Original-Schreiben $B$ [eati] $D$ [omi]ni Phil[ippi] Melanchtonis auf der Seite der Adressierung von einer Hand des 17. Jahrhunderts. Am unteren Blattrand wurde Num[ero] 24. verzeichnet; eine vergleichbare Notiz findet sich auch auf dem Brief Melanchthons an Matthäus Alber von 1534 (s. Nr.17).

14. Cod. theol. et philos. $2^{\circ} 79 \mathrm{c}$, fol. $308^{\mathrm{r}}$ : In einem Band der Abschriftensammlung des Helmstedter evangelischen Theologen, Orientalisten, Bibliothekars und Kirchenhistorikers Hermann von der Hardt (1660-1746) zum Konzil von Basel ${ }^{63}$, der wohl ursprünglich aus verstreut liegende Aktenbüschel bestand, die vermutlich erst zu Beginn des 20. Jahrhunderts in ungeordneter Reihenfolge gebunden wurde, findet sich (zusammen mit Nr.15 und 18) auch dieses Autograph Melanchthons, das sicher nur zufällig in diese Sammlung geraten ist. Melanchthon notierte hier den Anfang eines Konzepts von Ende August bzw. Anfang September 1530, das er allerdings abbrach und das Blatt schließlich für das Konzept der Articuli de quibus non convenit nobis cum adversariis verwendete (s. Nr. 16) ${ }^{64}$.

Die gesamte Abschriftsammlung Hermann von der Hardts zum Basler Konzil wurde 1786 für 1.000 Reichstaler von Herzog Carl Eugen von Württemberg (1728-1793) auf einer Bibliotheksreise durch Norddeutschland aus der Privatbibliothek Hermann von der Hardts für die Herzogliche Öffentliche Bibliothek gekauft ${ }^{65}$; diese Privatbibliothek war Teil des Nachlasses des Neffen Hermann von

${ }^{62}$ MBW T3, S. 604f. (Nr. 826); Regest: MBW 1, S. 354 (Nr. 826). - Krekler, Die Autographensammlung (wie Anm.3) S. 323. - Zu Matthäus Alber vgl. den fundierten Überblick bei Hans-Christoph Rublack, Art. Alber, Matthäus (1495-1570), in: Theologische Realenzyklopädie, hg. von Gerhard Krause/Gerhard Müller, Bd.2, Berlin/New York 1978, S. 170-177; zu Johann Butzbach und Martin Reiser vgl. Briefwechsel der Brüder Ambrosius und Thomas Blaurer 1506-1548, bearb. von Traugott Schiess, Bd.1, Freiburg i. Br. 1908, S. 145 Anm. 3 und 4.

${ }^{63}$ Dazu ein erster Überblick in: Carsten Kottmann, Zur Katalogisierung der theologischen Folio-Handschriften der Neuzeit in der WLB, in: WLB-forum 16,1 (2014) S. 38-41, hier S. 40 f. Vgl. zukünftig: Kottmann, Codices theologici (wie Anm.38).

${ }^{64}$ MBW T4/2, S. 625 (Nr. 1056); Regest: MBW 1, S. 436 (Nr. 1056).

${ }^{65}$ Christoph Friedrich StäLIN, Zur Geschichte und Beschreibung alter und neuer Büchersammlungen im Königreich Württemberg, insbesondere der Königlichen öffentlichen Bibliothek in Stuttgart und der mit derselben verbundenen Münz-, Kunst- und Alterthümersammlung, Stuttgart/Tübingen 1838, S.6f.; Peter Amelung u. a., Art. Württembergische Landesbibliothek, in: Handbuch der historischen Buchbestände in Deutschland, hg. von Wolfgang KeHr, Bd. 8, Hildesheim 1994, S. 282-319, hier S. 283. 
der Hardts, Anton Julius von der Hardt (1707-1785) ${ }^{66}$. Die darin enthaltenen Melanchthon-Autographe stammen wohl aus der Reformationssammlung Hermann von der Hardts ${ }^{67}$; wie dieser im Einzelnen an die Stücke seiner Sammlung gelangte, ist jedoch unbekannt.

15. Cod. theol. et philos. $2^{\circ} 79 \mathrm{c}$, fol. $306^{\mathrm{r}}-308^{\mathrm{v}}$ : Es handelt sich um das Konzept der Articuli de quibus non convenit nobis cum adversariis vom 3. September 1530, mit Nachträgen von der Hand Georg Spalatins ${ }^{68}$, und ist in direkter Verbindung mit den Bemühungen Melanchthons um die Confessio Augustana auf dem Augsburger Reichstag $1530 \mathrm{zu}$ sehen. Die Blätter weisen vor allem an den Rändern vereinzelt Wasserschäden auf.

Das Autograph stammt aus der Sammlung des Hermann von der Hardt zum Basler Konzil (s. Nr.14).

16. Cod. hist. $\mathbf{2}^{\circ} \mathbf{8 8 9 - 2 6}$, fol.38 ${ }^{\text {rv }}$ : Melanchthons Brief an den Reutlinger Reformator Matthäus Alber (1495-1570), geschrieben am 23. August 1530 von den Verhandlungen auf dem Augsburger Reichstag, ist ebenfalls mit der Frommann'schen Autographensammlung überliefert ${ }^{69}$. Das Blatt ist an der rechten oberen Ecke unter Textverlust beschädigt. Dieser Defekt kann erst nach 1717 aufgetreten sein, da Georg David Beger den Brief nach dem Autograph ohne Hinweis auf die Beschädigung herausgab ${ }^{70}$. Die verso-Seite enthält den Zusatz Original-Schreiben

${ }^{66}$ Der Auktionskatalog ist erhalten: Catalogus Bibliothecae D. Antonii Iulii von der Hardt Abbatis Michaelsteinensis, Theologiae Et LL. OO. Professoris Publ. Ordin.... Quae Codicibus Manuscriptis Et Libris Impressis ... Inter Quos Rarissimi Deprehenduntur, Constat In Aedibus b. Possessoris A. D. I August. Etc. Anni MDCCLXXXVI Publica Auctionis Lege Divendendae, Helmstedt: Schnorr, 1786, Praefatio und S. 24 f. (Nr. 110-112) (VD18 1061706X; Digitalisat unter http://vd18.de/de-sub-vd18/content/titleinfo/11992496, 18.09.2016).

${ }^{67}$ Vgl. Gustav A. E. Bogeng, Die großen Bibliophilen. Geschichte der Büchersammler und ihrer Sammlungen, Bd.3, Leipzig 1922, ND Hildesheim 1984, S. 117.

${ }_{68}$ MBW T4/2, S. 631 -634 (Nr. 1061); Regest: MBW 1, S. 437 f. (Nr. 1061). Vgl. auch Wilhelm Gussmann, Ein Melanchthonfund, in: Archiv für Reformationsgeschichte 23 (1926) S. 269-286, hier S.274f., 269f., 272-274. Vgl. Freiheit - Wahrheit - Evangelium (wie Anm.36) S. 96 f., und zukünftig: Kottmann, Codices theologici (wie Anm.38).

${ }^{69}$ MBW T4/2, S. 580-582 (Nr. 1037); Regest: MBW 1, S. 429 (Nr. 1037). - KreKLER, Die Autographensammlung (wie Anm.3) S.323. - Zu Matthäus Alber vgl. Rublack (wie Anm. 62).

70 [Georg David BEGER], Umbständliche Relation, wie es mit der Reformatione der Stadt Reuttlinge, so wohl vorinn und nach dem Jahr M.D.XVII. eigentlich hergegangen. Als in welchem Jahr der bewährte Rüstzeug Gottes Martinus Lutherus, den ersten Gott-geheiligt-allgemeinen Anfang gemacht. Mit vielen uralten Original-Documentis, [Reutlingen]: Johann Georg Füsing, 1717, S.199-201. - Die Abschrift des Autographs in HStAS J 1 Nr.127, Bd.4, fol.227v $-228^{r}$ (ca. Mitte 16. Jh.) weist ebenfalls keinen Textverlust aufgrund der Beschädigungen auf. 
$B$ [eati] $D$ [omi]ni Phil[ippi] Melanchtonis von einer Hand des 17. Jahrhunderts (s. Nr.13 und 17), sowie am unteren Rand den Rest eines Siegels mit dem zeitgenössischen Vermerk von anderer Hand: Scriptus [?] a[nno] [15]30.

17. Cod. hist. $2^{\circ} 889-26$, fol.37rv: Auch dieser Brief Melanchthons ging an den Reutlinger Reformator Matthäus Alber; er wurde an einem unbekannten Tag im Jahr 1534 in Wittenberg geschrieben und geht auf eine Kritik an den Reutlinger kirchlichen Verhältnissen ein ${ }^{71}$. Dieser Brief stammt ebenfalls aus der Frommann'schen Autographensammlung ${ }^{72}$. Auf der recto-Seite findet sich neben der Adressierung der Eintrag Original-Schreiben $B$ [eati] $D$ [omi]ni Phil[ippi] Melanchtonis von einer Hand des 17. Jahrhunderts, und am unteren Blattrand wurde von anderer Hand die Jahreszahl 1534, und darunter von einer weiteren Hand Num[ero] 35. geschrieben (s. Nr. 13 und 16).

18. Cod. theol. et philos. $2^{\circ} 79 \mathrm{c}$, fol. $314^{\mathrm{r}}-319^{\mathrm{r}}$ : Philipp Melanchthons Gutachten für (wahrscheinlich) Kurfürst Johann Friedrich I. von Sachsen, genannt Friedrich der Großmütige (1503-1554) stammt wohl von Dezember 1536 und wurde anlässlich des geplanten Bundestages am 7. Februar 1537 in Schmalkalden über das von Papst Paul III. vorgesehene Konzil von Mantua erstellt ${ }^{73}$. Auch dieses Autograph wurde wohl versehentlich der Abschriftensammlung des Hermann von der Hardt zum Basler Konzil zugeordnet und schließlich in diesen Band eingebunden (s. Nr. 14).

19. Cod. hist. $2^{\circ} 889-26$, fol.40r $-41^{\text {v }}$ : Melanchthon schrieb den Entwurf eines Briefs des Wittenberger Studenten Caspar Beyer an dessen Onkel, den brandenburgisch-ansbachischen Kanzler Sebastian Heller († 1542). Der undatierte Entwurf, überschrieben mit Epistola scripta manu D[octoris] Philippi Melanchthonis von einer Hand des 18. Jahrhunderts, muss zwischen 1537 und 1542 entstanden $\operatorname{sein}^{74}$. Melanchthon hat mindestens ein weiteres Mal ein Brief Beyers an Heller entworfen ${ }^{75}$. Beigefügt ist eine Abschrift des Briefentwurfs aus dem 18. Jahrhunderts; beides stammt aus der Frommann'schen Autographensammlung ${ }^{76}$.

${ }^{71}$ MBW T6, S. 263 f. (Nr. 1516); Regest: MBW 2, S. 165 (Nr. 1516).

72 Krekler, Die Autographensammlung (wie Anm.3) S. 323.

73 MBW T7, S. 307-310 (Nr. 1826, nach dieser Hs.); Regest: MBW 2, S. 287 f. (Nr. 1826); Gussmann (wie Anm. 68); zukünftig: Kottmann, Codices theologici (wie Anm. 38). - Zum geplanten Bundestag vgl. Gabriele Haug-Moritz, Der Schmalkaldische Bund 15301541/42. Eine Studie zu den genossenschaftlichen Strukturelementen der politischen Ordnung des Heiligen Römischen Reiches Deutscher Nation (Schriften zur südwestdeutschen Landeskunde, Bd. 44), Leinfelden-Echterdingen 2002, S. 60.

74 Regest: MBW 9, S. 31 (Nr. 9335). - Zu Beyer und Heller vgl. MBW 9, 130 f. (Nr. 2884 a).

75 MBW T8, S. 493 f. (Nr.2244 a); Regest: MBW 9, S. 116 (Nr.2244 a).

76 Krekler, Die Autographensammlung (wie Anm.3) S. 324. 
20. Cod. hist. $\mathbf{2}^{\circ} \mathbf{8 8 9 - 2 6}$, fol. $44^{\mathrm{r}}-\mathbf{4 5}^{\mathrm{v}}$ : Am 7. September 1538 (oder kurz danach) schrieb ein unbekannter Student einen Brief an einen unbekannten Empfänger; zu diesem Brief schrieb Melanchthon Korrekturen und einen unvollendeten Alternativentwurf an den Rand von fol. $44^{\mathrm{r}}$ und auf fol. $45^{\mathrm{rv}} .^{77}$ Friedrich Wilhelm Frommann, aus dessen Autographensammlung der Brief stammt, fertigte eine Abschrift von Melanchthons Alternative $\mathrm{an}^{78}$.

21. Cod. hist. $4^{\circ}$ 637: 1964 kaufte die WLB über die Parke-Bernet Galleries in New York zwei autographe Briefe Melanchthons aus der Bibliothek des Arne Pettersen (Tuckahoe, NY) für 1.100 bzw. $600 \mathrm{DM}^{79}$; für diese Erwerbungen wurde ein Antrag auf zusätzliche Mittel bei der Volkswagenstiftung Hannover gestellt ${ }^{80}$. Einen Brief schrieb Melanchthon am 28. März 1545 auf deutsch an den Rat der Stadt Breslau; dieser Brief stammt auch ursprünglich aus der Stadtbibliothek Bres$\mathrm{lau}^{81}$. Den zweiten Brief vom 13. Juli 1549 schrieb Melanchthon in lateinischer Sprache an den Philologen und Pädagogen Esrom Rüdinger (1523-1591) in Leipzig $^{82}$. Beide Briefe befanden sich im Besitz des lutherischen Theologen Georg Theodor Strobel (1736-1794), der angab, die beiden erhaltenen Briefe Melanchthons an Rüdinger „in autographo“ zu besitzen ${ }^{83}$. Den beiden Briefen liegt ein illustriertes Flugblatt bei („Epitaphium Des Ehrwirdigen Hochgelarten Theuren Mannes, Herrn Philippi Melanchtonis, Leipzig: Sturmer, 1560“), das bisher nicht nachgewiesen ist.

22. R 16 Lut 1: In diesem Band, der unter den Luther-Autographen bereits ausführlich beschrieben wurde (s. Nr.10), findet sich auch auf der Vorder- und Rückseite des Vorsatzblattes ein Autograph Philipp Melanchthons, eine deutsche Übersetzung von Phil 2,13-16 $6^{84}$.

77 MBW T8, S.212f. (Nr. 2090a); Regest: MBW 9, S.112 (Nr.2090a).

78 Cod. hist. ${ }^{\circ}$ 889-26, fol. 43 rv. Vgl. KreKLer, Die Autographensammlung (wie Anm.3) S.324.

79 Mss \& early printed books. A large group of works by and about Martin Luther; Reformation tracts in German and English. Four pages of MS by Luther on the Sacrament. Three important XIII Century MSS. The Augsburg Josephus of 1470, finely illuminated. Federmann's “Indianische Historia," 1557. From the Library of Arne Pettersen, Tuckahoe, New York, sold by his order, Public Auction Tuesday and Wednesday, November 10 and 11 ... Parke-Bernet Galleries Inc., New York 1964, S. 86 (Nr. 426 und 429).

${ }^{80}$ Vgl. Niedersächsisches Landesarchiv Hannover, V.V.P. 74 Acc. 74/97 Nr.187.

${ }^{81}$ MBW T14, S. 232 (Nr. 3865); Regest: MBW 4, S. 207 (Nr. 3865). - Signatur der Provenienz: Stadtbibliothek Breslau, Briefbd. 15 Nr. 58.

82 CR 7, S. 435 f. (Nr. 4563); Regest: MBW 5, S. 500 (Nr. 5594).

83 Georg Theodor Strobel, Neue Beyträge zur Litteratur, besonders des sechszehnten Jahrhunderts. Freunden der Kirchen-, Gelehrten- und Bücher-Geschichte gewidmet, Bd.2/1, Nürnberg/Altdorf 1791, S.12-14.

${ }^{84}$ Sснотt (wie Anm. 51) S. 163; Albrecht (wie Anm. 34) S. 275 und Anm.1. 
23. Cod. hist. $2^{\circ} 234$ a, I, Nr.5: Dier hier überlieferte Brief Melanchthons an Friedrich Staphylus in Königberg stammt vom 7. Oktober $1546^{85}$. Neben der Adresse wurde von späterer Hand hinzugefügt: Philippi Melanchton[is] literae ad Frid[ericum] Staphylum, sowie darunter: 7. Octobr[is]. Eine weitere spätere Hand fertigte auf der recto-Seite links nebem dem Brieftext eine unvollendete Transskription an, die mit dem Hinweis Ep[isto]la $I^{\mathrm{a}}$. abschließt.

Der Brief befand sich im Besitz des Theologieprofessors am Ulmer Gymnasium und Ulmer Münsterpredigers Albrecht Frick (1714-1776), der ihn Johann Georg Schelhorn (1694-1773) schenkte ${ }^{86}$. Wie der Brief schließlich in die Herzogliche Öffentliche Bibliothek gelangte, ist nicht bekannt; Verbindungen zwischen Schelhorn und Herzog Carl Eugen von Württemberg sind aber belegt ${ }^{87}$, so dass davon ausgegangen werden kann, dass Carl Eugen den Melanchthon-Brief in die Bibliothek einbrachte.

24. Cod. theol. et philos. $2^{\circ}$ 346: Dieses Gebet, das unter anderem das Treffen von Maria und Elisabeth (Lk 1,39-45) erwähnt, wurde wahrscheinlich für den Tag Marï̈ Heimsuchung (2. Juli) geschrieben. Das einseitige Konzept dürfte ca. 1547 entstanden sein; da weder der Text nähere Hinweise auf eine Entstehungszeit aufweist, noch das verwendete Papier ein Wasserzeichen enthält, lässt sich das Gebet wohl nicht näher datieren. Allerdings wird von Melanchthon in einem Brief an Benedikt Schumann vom 2. Juli 1547 das Treffen (synodus) zwischen Maria und Elisabeth dem Konzil (synodus) von Trient gegenüber- und positiv vorangestellt ${ }^{88}$ - in diesem Zusammenhang könnte das Gebet stehen. Die Handschrift wurde 1958 beim Auktionshaus J. A. Stargardt in Berlin für 250 DM gekauft ${ }^{89}$.

25. Cod. hist. $\mathbf{2}^{\circ} \mathbf{8 8 9 - 2 6}$, fol. $47^{\mathrm{rv}}$ : Ebenfalls aus der Frommann'schen Autographensammlung stammt der Brief Melanchthons an den Königsberger Theologieprofessor Friedrich Staphylus (1512-1564), geschrieben in Wittenberg am 20. August $1547^{90}$. Parallel zum eigenhändigen Brieftext Melanchthons wurde am Blattrand eine Transkription wohl des 18. Jahrhunderts eingetragen. Zusätzlich wurde auf der verso-Seite das Abfassungsdatum des Briefs (20. August[i] 1547

${ }^{85}$ MBW T15, S. 466 (Nr. 4404 a); Regest: MBW 4, S. 427 (Nr. 4404).

${ }^{86} \mathrm{Zu}$ Frick vgl. Ulrich Horst, Die Rechtsgelehrtenfamilie Wick aus Ulm a.D., in: Genealogie 13 = 26 (1977) S. 561-572, 605-617, hier S. 605; zu Schelhorn vgl. ADB 30 (1890) S. $756-759$.

${ }^{87} \mathrm{Vgl}$. Eberhard Zwınk, Die Stuttgarter 36-zeilige Bibel. Exemplarspezifische Merkmale und Aufhellung des Provenienzgangs, in: WLB-forum 12,1 (2010) S.2-19, hier S. 14.

${ }^{88}$ MBW T17, S. 27 f. (Nr. 4793); Regest: MBW 5, S. 134 (Nr. 4793).

${ }^{89}$ Autographen. Auktion am 13. Mai 1958 in Marburg ... (J.A. Stargardt, Katalog 537), Marburg 1958, S. 121.

${ }^{90}$ MBW T17, S.113f. (Nr.4852); Regest: MBW 5, S.161 (Nr.4852); CR 6, Nr.3979; zukünftig: Kottmann, Codices theologici (wie Anm.38). - Krekler, Die Autographensammlung (wie Anm.3) S.323 f. 
$v$ [el] 48) vermerkt. Auf fol. $46^{\text {rv }}$ ist eine weitere Transskription des Briefs beigefügt, dieses Mal von der Hand Friedrich Wilhelm Frommanns und überschrieben mit: Literae autographae Philippi Melanchtonis professoris tunc Witebergensis, ad Fridericum Staphylum. Frommann hatte hasce literas von dem Mönch und Bibliothekar des Benediktinerklosters Ottobeuren Michael Reichbeck am 9. Mai 1753 erhalten $^{91}$. Offensichtlich hatte Frommann ursprünglich mehrere Briefe Melanchthons an Friedrich Stapylus gesammelt, von denen heute nur noch dieser eine erhalten ist. Die Briefe sind mit der Büchersammlung des 1553 zum katholischen Glauben konvertierten Staphylus, am Ende seines Lebens Professor in Ingolstadt, wohl bereits 1616 als Vermächtnis des Sohnes Friedrich Staphylus jun. in die Klosterbibliothek nach Ottobeuren gelangt ${ }^{92}$.

26. Bb deutsch 154502: Diese Ausgabe einer deutschen Lutherbibel, gedruckt 1545 bei Hans Lufft in Wittenberg ${ }^{93}$, enthält neben autographen Einträgen von Johannes Bugenhagen (1485-1558) vom 3. März 1548, von Georg Major (15021574) von 1548, von Sebastian Kugler von 1579 und einer gefälschten (kopierten) Spruchauslegung Luthers von $1546^{94}$ einen undatierten Eintrag Philipp Melanchthons zu Joh 14,23 mit dem griechisch geschriebenen Bibelvers und einer lateinischen Auslegung.

Der Band stammt aus der Bibelsammlung des Kopenhagener Pfarrers Josias Lorck (1723-1785) und ist mit dem Besitzeintrag J[osias] Lorck Pastor Hafniensis gekennzeichnet. Herzog Carl Eugen von Württemberg 1784 kaufte diese Sammlung auf seiner Reise nach Niedersachsen und Dänemark für die Herzogliche Öffentliche Bibliothek ${ }^{95}$. Ein weiterer Besitzeintrag auf dem vorderen Spiegel sowie auf dem Titelblatt verweist auf Johann Albrecht Mey und das Jahr 1710; von

${ }^{91} \mathrm{Zu}$ Reichbeck vgl. Ulrich FausT, Ottobeuren, in: Die Männer- und Frauenklöster der Benediktiner in Bayern, bearb. von Michael Kaufmann u.a., Bd. 2 (Germania Benedictina, Bd. II/2), St. Ottilien 2014, S.1541-1609, hier S. 1588.

92 Vgl. Paul Lehmann, Aus der Bibliothek des Reformators Johannes Hessius, in: Aus der Welt des Buchs. Festgabe zum 70. Geburtstag von Georg LeYH (Zentralblatt für Bibliothekswesen, Beiheft 75), Leipzig 1950, S. $101-124$.

${ }_{93}$ Biblia: das ist: Die gantze Heilige Schrifft: Deudsch Auffs new zugericht. D. Mart. Luth. Begnadet mit Kurfurstlicher zu Sachsen Freiheit, Wittenberg: Hans Lufft, 1545 (VD16 B 2717; VD16 B 2718). Der Druck ist digitalisiert: http://digital.wlb-stuttgart.de/ purl/bsz351733388 (18.09.2016). Vgl. Deutsche Bibeldrucke 1466-1600, beschrieben von Stefan Strohm unter Mitarbeit von Peter Amelung/Irmgard Schauffler/Eberhard Zwink (Die Bibelsammlung der Württembergischen Landesbibliothek Stuttgart, Bd.2,1), Stuttgart-Bad Cannstatt 1987, S. 202 (Nr. E 350).

${ }^{4} \mathrm{Vgl}$. WA 48, S. 136.

95 Vgl. Christian Herrmann, Bibelsegen und Bibelfleiß. Die Sammlung Lorck, in: Carl Eugens Erbe. 250 Jahre Württembergische Landesbibliothek. Eine Ausstellung der Württembergischen Landesbibliothek aus Anlass ihrer Gründung am 11. Februar 1765 vom 11. Februar bis 11. April 2015, hg. von Vera Trost in Zusammenarbeit mit Hans-Christian Pust, Stuttgart 2015, S.78-83. 
diesem könnte Lorck den Druck erhalten haben. Zu Mey ist jedoch nichts bekannt ${ }^{96}$.

27. Im Bestand der WLB nicht erhalten hat sich ein Brief Melanchthons an Bischof Otto von Augsburg vom 17. Februar 1552 ${ }^{97}$. Dieser Brief befand sich $1753 / 1754$ in der württembergischen Kunstkammer (Schreiben eigenhandiges Philippi Melanchtonis $d[\mathrm{e}] d[\mathrm{it}]$ Nüremberg 17. Feb[ruarii] a[nn]o 1552 $)^{98}$ sowie 1776 in der Bibliothek des württembergischen Naturalienkabinetts ${ }^{99}$, von wo sie im gleichen Jahr in die damalige Herzogliche Öffentliche Bibliothek gelangte. Eine Abschrift, die nach diesem verlorenen Autograph angefertigt worden sein könnte, befindet sich heute im Hauptstaatsarchiv Stuttgart ${ }^{100}$.

28. Cod. Don. 898, fol.167 : Im Stammbuch des aus dem österreichischen Hallein stammenden Daniel Prasch (1561 - 1630) findet sich ein Eintrag, dessen Zuordnung zu Melanchthon allerdings fraglich ist - es könnte sich auch um eine sehr sorgfältige Nachahmung handeln ${ }^{101}$. Dieser vermeintliche Melanchthon-Eintrag stammt wohl zusammen mit Autographen von Philipp Melanchthon d.J. und Paul Eber aus dem Besitz des Vaters, Georg Prasch (1524-1592), der neben Luther auch Melanchthon in Wittenberg hörte ${ }^{102}$. Das fragliche Melanchthon-Autograph ist eine Precatio bestehend aus zwei Distichen - die erste hatte Melanchthon erstmals in einem Brief an Georg Cracow in Stettin vom 1. Januar 1553 geschrieben, danach wieder am 23. April 1553 an Johannes Matthesius in Joachimsthal und am 1. März

${ }^{96}$ Eine Verwandtschaft mit dem bei Hans BöscH, Geschwornenbuch der Nürnberger Barbierer und Wundärzte, in: Mitteilungen aus dem germanischen Nationalmuseum 1893, S. 29-39 genannten Johann Albrecht Mey (1633-1688) ist denkbar.

97 Regest: MBW 6, S.269 (Nr.6351). Die Vorlage für die Edition befindet sich heute in Modena, Archivio di Stato, Concilio di Trento; vgl. Georg Kupke, Drei unbekannte Melanchthon Briefe, in: Quellen und Forschungen aus italienischen Archiven und Bibliotheken 2 (1899) S. 317-320, hier S. 320.

98 HStAS A 20 a Bü 37, $7^{\mathrm{r}}$ [4 ${ }^{\mathrm{r}}$; ; vgl. auch HStAS A 20 a Bü 87, pag. 14.

99 WLB Stuttgart, Bibliotheksakten Nr. 14; HStAS A 20 a Bü 113. Vgl. Kottmann, Die Bibliothek (wie Anm. 22).

100 HStAS A 63 Bü 8/23.

101 Stammbücher bis 1625, beschrieben von Ingeborg KREKLER (Die Handschriften der Württembergischen Landesbibliothek Stuttgart, Sonderreihe 3), Wiesbaden 1999, S. 80 (Nr. 24); Wolfgang Klose, Corpus alborum amicorum. CAAC. Beschreibendes Verzeichnis der Stammbücher des 16. Jahrhunderts (Hirsemanns biographische Handbücher, Bd.8), Stuttgart 1988, S. 121; Karl August Barack, Die Handschriften der Fürstlich-Fürstenbergischen Hofbibliothek zu Donaueschingen, Tübingen 1865, ND Hildesheim/New York 1974, S.597. - Digitalisat unter: http://digital.wlb-stuttgart.de/purl/bsz420412670/page/333 (18.09.2016). - Die Zweifelhaftigkeit der Authentizität laut E-Mail von Dr. Christine Mundhenk (Heidelberg) vom 05. 09.2016.

102 Johann Seifert, Stamm-Taffeln Gelehrter Leute, Nach Ordnung des Alphabets ..., Bd. 2, Regensburg 1723, Nr. 27 A. 
1554 an Simon Haliaeus in Kaaden, allerdings jeweils mit abweichender Fortsetzung $^{103}$. Der Eintrag ist auf einem marmorierten Papier geschrieben, das an den Rändern mit einer gedruckten Zierleiste versehen wurde. Auf fol. $167^{\mathrm{r}}$ und bereits auf fol.166 ${ }^{\mathrm{r}}$ (dem Autograph Philipp Melanchthons d.J.) finden sich KuperstichBildnisse von dem Vater Melanchthon ${ }^{104}$. Wie das Stammbuch in die FürstlichFürstenbergische Hofbibliothek Donaueschingen kam, ist nicht bekannt; 1994 gelangte sie dann mit einem Teil des Donaueschinger Handschriftenbestands in die Württembergische Landesbibliothek ${ }^{105}$.

29. Bb deutsch 155603: Es handelt sich um eine Ausgabe der Lutherbibel, gedruckt von Hans Lufft, Wittenberg, 1556 ${ }^{106}$. Im ersten Band dieser Ausgabe wurde auf den hinteren Spiegel ein Blatt mit einem Gedicht Philipp Melanchthons von 1554 eingeklebt: De vagiente ecclesia ${ }^{107}$. Von späterer Hand wurde hinzugefügt: Scriptum manu D[octoris] Philippi Melanthon[is] 1554. Neben Melanchthons Autograph finden sich in den beiden Bänden auch ein Eintrag von Johannes Bugenhagen (1485-1558; vorderer Spiegel des ersten Bandes) sowie neben anderen Zitate von Lukas Osiander (1534-1604), Nikolaus Selneccer (1530-1592) und Johannes Caesarius (1468-1550).

Die Ausgabe enthält den Besitzvermerk Barbara Ditz sowie Kauf- und Bindevermerke. Sie stammt aus der Bibelsammlung des Nürnberger Theologen und Bibliographen Georg Wolfgang Panzer (1729-1805), die Herzog Carl Eugen von Württemberg 1786 kaufte $^{108}$.

30. Cod. hist. $2^{\circ}$ 527: Die Sammelmappe, die bereits unter den Luther-Autographen beschrieben wurde (s. Nr.11), enthält vier eigenhändige Briefe Melanchthons ${ }^{109}$. Diese Briefe stehen in direktem Zusammenhang mit der Reformation in Württemberg: Ein Brief ist adressiert an Erhard Schnepf in Stuttgart (5. Januar $1544)^{110}$ sowie drei Briefe an Johannes Brenz, ebenfalls jeweils in Stuttgart (18. Juni

103 CR 10, S. 618 (Nr.268); MBW 7, S.17 (Nr.6692.3); des Weiteren in MBW 7, S.60 (Nr.6804.2) und S. 164 (Nr.7097.1).

104 Krekler, Stammbücher bis 1625 (wie Anm. 101) S. 77 f., 80.

105 Vgl. Felix Heinzer, Die neuen Standorte der ehemals Donaueschinger Handschriftensammlung, in: Scriptorium 49 (1995) S.312-319.

106 Biblia: Das ist: Die gantze heilige Schrifft Deudsch. Doct. Mart. Luther, Wittenberg: Hans Lufft, 1556 (VD16 B 2740). Digitalisat unter: http://digital.wlb-stuttgart.de/purl/ bsz351746609 (18.09.2016); vgl. Deutsche Bibeldrucke 1466-1600 (wie Anm.93) S.229 (Nr.E 393).

107 CR 10, S. 624 (Nr. 280) (nicht nach diesem Autograph).

108 Georg Wolfgang Panzer, Entwurf einer vollständigen Geschichte der deutschen Bibelübersetzung D. Martin Luthers vom Jahr 1517 an, bis 1581, Nürnberg 1783, S. 426 f.; LÖFfler, Geschichte (wie Anm.1) S.26f.

${ }_{109} \mathrm{Vgl}$. Die historischen Handschriften der Königlichen öffentlichen Bibliothek, Bd.1 (wie Anm. 55) S.224.

110 MBW T13, S.34 (Nr. 3426); Regest: MBW 4, S. 16 (Nr.3426). 
$1555^{111}$; 21. April 1557112; 9. Februar 1559113). Die Briefe stammen aus dem Benediktinerkloster Weingarten und gelangten nach $1810 \mathrm{im}$ Zuge der Säkularisation in die damalige Königliche Öffentliche Bibliothek.

31. Cod. hist. $2^{\circ} 889-26$, fol. $32^{\mathrm{r}}-35^{\mathrm{v}}$ : Zusammen mit Johannes Brenz verfasste Melanchthon auf dem Wormser Religionsgespräch einen Brief an Herzog Christoph von Württemberg mit Datum des 28. November 1557, in dem sie um Unterstützung für den italienischen Humanisten Lelio Sozzino (1525-1562) bitten, der den Brief auch nach Stuttgart überbracht hatte ${ }^{114}$. Der Brief, geschrieben von Melanchthon und mitunterzeichnet von Brenz, ist in der Autographensammlung Frommann überliefert.

32. Phil.oct.6940: Dieser Band (Johann Boeschenstein, Hebraica grammaticae institutiones, Wittenberg: Johann Rhau-Grünenberg, 1518 [VD16 B 6372]), beinhaltet ebenfalls ein Melanchthon-Autograph. Allerdings ist dieser Band in der WLB seit 1972 vermisst; Details zum Autograph sind unbekannt.

33. Cod. hist. $4^{\circ}$ 95: Dieses Konvolut, das ebenfalls bereits unter den LutherAutographen beschrieben wurde (s. Nr. 4), überliefert neben ursprünglich mehreren Originalbriefen Luthers und einem autographen Brief des Tübinger Gräzisten Martin Crusius auch wohl einen Entwurf Melanchthons zu einer Abhandlung, die sich mit der Thematik der Rechtfertigung und Liebe befasst ${ }^{115}$. Die Entstehungszeit dieses Stücks ist nicht bekannt.

1753/1754 befand sich der Brief wohl in der württembergischen Kunstkammer (Handschrifft Philippi Melanchtonis in 2. Blättern) ${ }^{116}$ und wurde ab 1764 im Naturalienkabinett aufbewahrt, eine Abteilung der Kunstkammer (Philippe Melanchtonis Lateinische handschrifft, so aber fast unleserlich ist, auf einem Blatt in Folio, oder weil es in der Mitte zerrißen, auf 2. Blatt in 4to. $)^{117} .1776$ wurde der kleine Bibliotheksbestand des Naturalienkabinetts an die Herzogliche Öffentliche Bibliothek übergeben ${ }^{118}$.

111 Heinrich Ernst BindseIL, Philippi Melanchthonis epistolae, iudicia, consilia, testimonia aliorumque ad eum epistolae quae in Corpore Reformatorum desiderantur, Halle 1874, ND Hildesheim/New York 1975, S. 566f. (Nr. 571); Regest: MBW 7, S. 322 (Nr. 7525).

112 CR 9, S. 144 (Nr. 6234); Regest: MBW 8, S. 64 (Nr. 8202).

113 Bindseil (wie Anm. 111) S.442f. (Nr.452); Regest: MBW 8, S. 311 (Nr. 8850).

114 Regest: MBW 8, S. 157 (Nr. 8435). Der Brief ist bisher nicht gedruckt. - KreKLER, Die Autographensammlung (wie Anm.3) S. 324.

115 Die Historischen Handschriften der Königlichen öffentlichen Bibliothek, Bd.2 (wie Anm. 17) S.38f.; WA Br 14, S. 133.

116 HStAS A 20 a Bü 37, $7^{\mathrm{r}}$ [4 ${ }^{\mathrm{r}}$. Vgl. Kottmann, Die Bibliothek (wie Anm. 22) S. $595 \mathrm{u}$. 600 .

117 HStAS A 20 a Bü 87.

118 Vgl. WLB Stuttgart, Bibliotheksakten, Nr.14; HStAS A 20 a Bü 111; Löfrler, Geschichte (wie Anm.1) S. 15. 
34. Cod. hist. $2^{\circ}$ 889-26, fol.42r: Melanchthons undatierter lateinischer Spruch stammt aus der Sammlung Frommann; dort ist der Zettel $(13 \times 8 \mathrm{~cm})$ auf ein Blatt im Kanzleiformat neben ein gemaltes Portrait von Melanchthon geklebt. Der Spruch (Nulla possessio, nullae opes sunt durabiliores quam eruditio. Cetera enim bona omnia, quae maxime appetuntur in vita, multis casibus eripi nobis possunt.) ist darunter in einer Abschrift des 18. Jahrhundert wiedergegeben ${ }^{119}$. Das kleine Format des Zettels könnte darauf hinweisen, dass der Spruch ursprünglich auf dem Vorsatzblatt oder Ähnliches eines Drucks eingetragen und später aus dem Band herausgetrennt wurde. Manche Zeilen weisen auffällige Lücken auf, dabei könnte es sich um herausradierte, vielleicht original gestrichene Wörter handeln.

119 Krekler, Die Autographensammlung (wie Anm.3) S. 324. 